\title{
THE ORIGIN OF THE RS CVN BINARIES
}

\author{
P. BIE R M A N N \\ Astronomisches Institut, Universität Bonn, Bonn, F.R.G. \\ and \\ D. S. H A L L \\ Dyer Observatory, Vanderbilt University, Nashville, Tenn. U.S.A.
}

\begin{abstract}
We consider six possible origins for the RSCVn binaries based on the following possibilities. RS CVn binaries might now be either pre-main-sequence or post-main-sequence. A pre-main-sequence binary might not always have been a binary but might have resulted from fission of a rapidly rotating single pre-main-sequence star. The main-sequence counterparts might be either single stars or binaries.

To decide which of the six origins is possible, we consider the following observed data for the RSCVn binaries: total mass, total angular momentum, lack of observed connection with regions of star formation, large space density, kinematical age, and the visual companion of WW Dra. In addition we consider lifetimes and space densities of single stars and other types of binaries.

The only origin possible is that the RSCVn binaries are in a thermal phase following fission of a main-sequence single star. In this explanation the single star had a rapidly rotating core which became unstable due to the core contraction which made it begin to evolve off the main sequence. The present Be stars might be examples of such parent single stars.
\end{abstract}

\section{Introduction}

According to the recent review paper of Hall (1975a), the characteristics necessary for a binary to belong to the RSCVn group are the following: the orbital period is between one day and two weeks, the hotter component is $F$ or $G$ and $V$ or IV, and strong $\mathrm{H}$ and $\mathrm{K}$ emission is seen in the spectrum outside eclipse. The word 'strong' here means stronger than the normal emission observed in the $\mathrm{H}$ and $\mathrm{K}$ reversal as a result of the WilsonBappu effect in single stars. Based on this definition we know of the following $25 \mathrm{RSCVn}$ binaries: UX Ari, CQ Aur, SS Boo, SS Cam, RU Cnc, RS CVn, AD Cap, UX Com, RT CrB, WW Dra, Z Her, AW Her, MM Her, PW Her, GK Hya, RT Lac, AR Lac, RV Lib, VV Mon, LX Per, SZ Psc, TY Pyx, RW UMa, RS UMi, and HD 118216.

In addition, we provide the following list of 13 properties which are characteristic of the RSCVn binaries, but we point out that not all 25 binaries display all 13 properties. (1) The $\mathrm{H}$ and $\mathrm{K}$ emission arises from the cooler star (or from both stars). (2) The cooler star is around KO IV. (3) $\mathrm{H} \alpha$ is seen in emission. (4) A persistent nearly sinusoidal wave distorts the light curve outside eclipse, can be attributed to the cooler star, does not always have a constant amplitude, and can account for the changing depth of primary minimum and the changing displacement of secondary eclipse. (5) This wave migrates towards decreasing orbital phase, not always at a uniform rate. (6) There are sometimes irregular light variations in light not attributable to the migrating wave. (7) There is an ultraviolet excess in one or both components. (8) There is an infrared excess in one or both components. (9) Strong centimeter radio emission has been observed in three binaries: UX Ari RT Lac, AR Lac. (10) There are very large, irregular changes in orbital period that cannot be explained as apsidal motion or orbital motion around a third body. (11) In several cases these period variations are correlated with the migrating wave. (12) With 
only two exceptions, AD Cap and RT Lac, the mass ratio is very nearly unity. (13) Both components are detached from their Roche lobes.

Inquiry concerning the evolutionary status has proceded along the following tortuous path. Judging by absolute dimensions, Plavec and Grygar (1965) realized that the evolutionary status is somehow unique. Roxburgh (1966a) suggested that binaries with undersize subgiants are in pre-main-sequence contraction, but it seems now (Hall, 1974) that his sample contained only a few RSCVn binaries, the rest being quite typical Algol-type binaries for which inaccurate absolute dimensions had misled others into considering them detached. References related to the Roxburgh paper are Hall (1968), Field (1969), and Barnes (1974). Catalano and Rodono (1967) suggested that the cooler component of RS CVn is in pre-main-sequence contraction. Hall (1972) considered the relative merits of pre- and post-main-sequence interpretations for the RSCVn binaries as a group and concluded that the former was more attractive. Huang (1973) suggested that the RS CVn binaries, if pre-main-sequence and losing mass as Hall (1972) claimed, might be progenitors of the W UMa binaries. Montle (1973) derived a kinematical age which is not consistent with either a pre- or post- main-sequence interpretation. Oliver (1974) discussed the evolutionary problem in detail, explored the possibility that they might be related somehow to case $A B$ post-main-sequence mass transfer, but concluded that, at present, there is no really satisfactory solution. Ulrich and Popper (1974) suggest that the components are post-main-sequence stars observed during the period of hydrogen burning in a thick shell but before the onset of rapid mass transfer. Hall (1975b) showed that the F8 V visual companion to WW Dra makes a pre-main-sequence interpretation untenable.

In this contribution we make use of the following additional input data. The RSCVn space density is about $1 \times 10^{-6}$ systems $\mathrm{pc}^{-3}$ according to Montle (1973) or about $2 \times 10^{-6}$ systems $\mathrm{pc}^{-3}$ according to the list of Dworak (1973). These values are lower limits because both Montle and Dworak considered only eclipsing systems. The total systemic mass has a range of $1.75 M_{\odot}<M<2.98 M_{\odot}$. The kinematical age is about $1-3$ $\times 10^{8} \mathrm{yr}$ according to Montle. This age is relatively reliable because it was deduced from determinations of both the mean height above and below the galactic plane, $112 \pm 20 \mathrm{pc}$, and the dispersion of the velocity component perpendicular to the galactic plane, $10.0 \pm 2.5 \mathrm{~km} \mathrm{~s}^{-1}$. It is generally accepted that the RSCVn systems are not connected with nebulosity, associations or other regions of stellar formation, but we admit we can cite no first-hand research to support this claim. We take space densities for single mainsequence stars from Biermann and Tinsley (1974) and for binary stars from a variety of sources, for example, Kraft (1967).

\section{Six Possible Evolutionary Cases}

Most inquiry into the problem of the evolutionary status of the RSCVn binaries has been restricted to debate as to whether RSCVn binaries have evolved from or will evolve into main-sequence binaries. But we point out here that single stars must also be considered as possible progenitors or descendents. Also we must consider the possibility that a pre-main-sequence binary was not always a binary; it might have resulted from fission of a rapidly rotating single pre-main-sequence star. Thus we consider six logical possibilities for the RSCVn binaries. 
I. Pre-main sequence binaries now; become main-sequence binaries.

(a) always binary while pre-main-sequence.

(b) resulted from fission of a rapidly rotating single pre-main-sequence star.

II. Pre-main-sequence binaries now; become single stars on the main sequence.

(a) always binary while pre-main-sequence.

(b) resulted from fission of a rapidly rotating single pre-main-sequence star.

III. Post-main-sequence binaries now; were binaries on the main sequence.

IV. Post-main-sequence binaries now; were single stars on the main sequence.

These six cases can be seen in perspective in the following way. Given that pre-stellar clouds of interstellar material should be characterized by a continuum ranging from large to small angular momentum per unit mass $(J / M)$, one should anticipate the following continuum of evolutionary possibilities. (1) $J / M$ so large that the pre-stellar cloud fissions before becoming a star. This would include cases Ia and IIa. (2) $J / M$ enough smaller that the cloud can become a star, but a star which fissions before reaching the main sequence. This would include cases $\mathrm{Ib}$ and $\mathrm{Ilb}$. (3) $J / M$ still smaller, enough so that the star can reach the main sequence without fissioning; but later, when its rapidly rotating helium-rich core begins to contract, the star fissions. This would correspond to case IV. (4) $J / M$ so small that the single star never fissions.

Now we consider these six cases in turn, drawing attention primarily to data or constraints which argue against their likelihood. These arguments are summarized in Table I.

\section{Case Ia}

In this case the RSCVn systems are pre-main-sequence binaries which evolve into mainsequence binaries. This case includes Huang's suggestion that the RS CVn binaries become W UMa binaries.

The very large space density of the RSCVn binaries argues against this case. This is because, counting both W UMa binaries and detached main-sequence binaries, we do not find nearly as many systems as we would expect on the main sequence if the RSCVn binaries really are pre-main-sequence.

Since the W UMa systems and the RSCVn systems embrace comparable ranges in mass, we would expect their space densities to be proportional to their ages. With the W UMa

TABLE I

Summary of arguments against various evolutionary interpretations for the RS CVn binaries

\begin{tabular}{|c|c|c|c|c|c|c|}
\hline \multirow{2}{*}{$\begin{array}{l}\text { present RS CVn binary: } \\
\text { main-sequence equivalent: }\end{array}$} & \multicolumn{4}{|c|}{ pre-main-sequence } & \multicolumn{2}{|c|}{ post-main-sequence } \\
\hline & \multicolumn{2}{|c|}{ binary system } & \multicolumn{2}{|c|}{ single star } & $\begin{array}{l}\text { binary } \\
\text { system }\end{array}$ & $\begin{array}{l}\text { single } \\
\text { star }\end{array}$ \\
\hline case: & Ia & $\mathrm{Ib}$ & IIa & IIb & III & IV \\
\hline \multicolumn{7}{|l|}{ argument: } \\
\hline space density & $x$ & $x$ & & & $?$ & \\
\hline kinematical age & $x$ & $?$ & $x$ & $?$ & $x$ & \\
\hline VW Draconis & $x$ & $?$ & $x$ & $?$ & & \\
\hline \multirow{2}{*}{$\begin{array}{l}\text { nebulosity, etc. } \\
\text { other }\end{array}$} & $x$ & $?$ & $x$ & $?$ & & \\
\hline & & & & $x$ & $x$ & \\
\hline
\end{tabular}


binaries thought to be evolving on a main-sequence time scale of $\sim 10^{9} \mathrm{yr}$, and the RSCVn binaries assumed here in this case to be evolving on a pre-main-sequence time scale of $\sim 10^{7} \mathrm{yr}$, the ratio would be around 100:1. But the observed ratio of their space densities is only unity or less. The W UMa space density, $10^{-6}$ systems $\mathrm{pc}^{-3}$ according to $\mathrm{Kraft}$, is perhaps less than the RSCVn space density, which must be greater than 1 or $2 \times 10^{-6}$ systems pc $^{-3}$.

If the RSCVn binaries evolve into detached main-sequence binaries, the latter should outnumber the former by the ratio of their respective ages: $3 \times 10^{9} \mathrm{yr} 10^{-7} \mathrm{yr}=300: 1$. In any given catalogue which is complete down to a certain limiting magnitude, this ratio should be around 100:1, since the predicted main-sequence counterparts should be around 0.5 to $1.0 \mathrm{mag}$ fainter. In the catalogue of Koch et al. (1963), there are 20 known RS CVn binaries. Within the same mass range and period range there are only 54 other binaries which could possibly be the predicted descendents. We excluded only known W UMa, Algol, and RSCVn systems, so the number 54 is an upper limit. Making a small correction to allow for the fact that fewer main-sequence binaries should be eclipsing, we conclude that the observed ratio is less than $4: 1$, which is significantly less than the above-mentioned 100:1. An analysis similar to this was first made by Oliver.

There are additional arguments against this case. Montle's kinematical age of $3 \times 10^{8}$ $\mathrm{yr}$ would rule out this pre-main-sequence interpretation. The less massive F8 V visual companion to WW Dra (Hall 1975b) proves that at least this particular RS CVn binary cannot be pre-main-sequence. And we recall the lack of observed association with nebulosity.

\section{Case Ib}

This case is the same as Ia except that the RSCVn binary is a pre-main-sequence object resulting from fission of a rapidly (differentially) rotating star just before it begins nuclear burning. Fission of this type was studied by Roxburgh (1966b) and by Bodenheimer and Ostriker (1970).

The total contraction time (contraction as a single star up to the point of fission, plus contraction of the two components of the binary system up to the main sequence) becomes longer than that in case Ia. The maximum total contraction time is $3-8 \times 10^{7}$ $\mathrm{yr}$ for an original single star of $2-3 M_{\odot}$, still shorter than the kinematical age of Montle, but not by much. In a similar manner there might not be a serious problem with WW Dra or with the lack of association with nebulosity, etc.

The large space density of the RSCVn binaries, however, remains a problem as it did for case Ia. Although the contraction times and hence the ages are longer (by a factor of around five) than in case Ia, we are dealing with parent stars which are less common, by about the same factor, due to the larger mass. Thus this case also would predict more main-sequence descendents, detached or W UMa binaries, than are observed.

\section{Case IIa}

In this case the RSCVn systems are pre-main-sequence binaries, but here we imagine they somehow evolve into main-sequence single stars.

This case does not have trouble with the large RSCVn space density as did both case Ia and $\mathrm{Ib}$, because there are appreciably more main-sequence single stars in this mass range than main-sequence binary stars in the same mass range and with the appropriate 
separations. But it has just as much trouble with the kinematical age, the visual companion to WW Dra, and the lack of association with nebulosity, etc.

\section{Case IIb}

This case is the same as case IIa except that, as with Ib, the pre-main-sequence RSCVn binary results from fission of a rapidly rotating pre-main-sequence single star.

Somewhat surprisingly, we cannot find any difficulty in reconciling this case with the large RSCVn space density. As with case IIa, the RSCVn space density presents no problem; and, with the longer total contraction times, the problems remaining with case Ila are not serious here.

But it is difficult to believe this case can really be true. The implication of this evolutionary case, if true, is that the two components of RSCVn binaries move closer together (Huang, 1973) as they approach the main sequence, make contact with each other to appear as W UMa binaries, and then merge completely to form single stars. What is difficult to accept is the last part of this sequence, because the W UMa binaries may not become main-sequence single stars but rather evolve into the U Gem binaries (Kraft, 1967). Recently it has been suggested that the W UMa binaries might evolve into single stars. But if they have lifetimes of about $10^{9} \mathrm{yr}$ they present problems for this case.

\section{Case III}

In this case the RSCVn systems are the result of post-main-sequence evolution of a binary originally on the main sequence. For the sake of completeness one should consider evolution both before and after the primary component first overflows its Roche lobe.

The chief difficulty with this case is that the RSCVn binaries simply do not fit into the post-main-sequence mass transfer theory for the evolution of close binaries. If one tries to fit them in to this theory, there are serious problems, which have been enumerated by Hall (1972) and by Oliver (1974). They could be explained as freak occurrences, but their large space density makes this impossible. Moreover, the kinematical age seems to rule out this type of post-main-sequence evolution because stars in this mass range would begin to leave the main sequence only after $10^{9}$ or $10^{10} \mathrm{yr}$.

We should point out that Montle's calibration of velocity dispersion versus age was based on data compiled by Delhaye (1965). If the newer calibration of Wielen (1974) is used, Montle's value of $10.0 \mathrm{~km} \mathrm{~s}^{-1}$ would indicate a considerably greater age, about $2.10^{9} \mathrm{yr}$. Such an age would favour a case III interpretation.

\section{Case IV}

In this case the RSCVn binaries are post-main-sequence objects resulting from the fission of a rapidly rotating main-sequence single star.

This last case is the only one except IIb which does not encounter and difficulties with the large RSCVn space density and therefore, if only by default, merits serious consideration. One possible difficulty is the question of whether the parent main-sequence single star is capable of containing the same total amount of angular momentum that, after fission, the resultant RSCVn binary will. If the original rotation law is of extreme form, i.e., the rotational velocity is nearly Keplerian throughout the outer, radiative, zone, then it seems that a single star is capable of this, provided its mass is around 4 or $5 M_{\odot}$. The 
only problem is that instabilities of the rotation laws are not completely understood, and might present problems.

\section{The Most Likely Case: Fission of a Single Star}

Since this last case appears the most attractive of the six, let us examine it in more detail. In this case a single rapidly rotating main-sequence star splits in the post-main-sequence phase and the dynamical process of fission puts the components in the thermal phase where they relax on thermal timescales to nuclear post-main-sequence phases. If breakup should occur in this manner, then the stellar material will be strongly disturbed, possibly resulting in a state where one or both components has equal entropy throughout the outer regions, or maybe the entire star, corresponding to the Hayashi phase. Hence one component or both should lie near the Hayashi track and the other, which is unlikely to have escaped some mixing, should lie at least somewhat above the main-sequence. Since the fission of evolved stars is not understood at present, this case has to remain speculative. Theoretically, the calculations of Kippenhahn et al. (1970) have shown that the core of a star contracts and speeds up its rotation in the post-main-sequence phase and may become unstable to fission. Whether the original main-sequence star can start off with sufficient angular momentum in its core, is a question of the instabilities of differential rotation and the transport of angular momentum (Goldreich and Schubert, 1967; Fricke, 1968; Kippenhahn, 1969; James and Kahn, 1970, 1971; Fricke and Kippenhahn, 1972); the relevant timescales of mixing turn out to be rather long in the case of local instability of a rotation law, on the order of the thermal timescale or longer. The competition between this local mixing and the large scale Eddington-Voigt circulation on the one hand and the buildup of the molecular weight barriers that inhibit mixing on nuclear time scales on the other hand is not clear at present.

Points in favor of this case are the following. (1) The main-sequence lifetime of the predicted parent single star agrees quite well with the kinematical age of Montle. (2) Of all six cases considered this one has the least trouble in accounting for the large RSCVn space density. (3) We know that stars come onto the main sequence with high rotation and we know that the incidence of close binaries is high among early-type main-sequence stars. Bodenheimer and Ostriker have shown that there need not be a break in the distribution of angular momentum over the different stars of the same mass. Thus it appears highly likely that some, if not many, stars come onto the main-sequence with marginal stability to breakup. (4) It is tempting to identify the main-sequence counterparts as the Be stars, which are known to be rotating rapidly and have the proper mass. (5) To the extent that the two components after fission are in a thermal phase, we are in a good position to account for the many observational peculiarities (gross sunspot activity, bright emission lines, strong solar-wind-type mass loss, radio emission, etc.) in a natural way. (6) As shown by Atkins and Hall (1972), the observed infrared excess along with the observed $\mathrm{K} 0$ spectral type indicates that the cooler component is on the Hayashi track, which is one expectation of this case. (7) Even the earlier component is in general somewhat above the main sequence, another expectation of this case.

It will be possible to devise a number of observational tests for this theory. Most obvious is the prediction that the ${ }^{7} \mathrm{Li}$ abundance be low; otherwise the RSCVn binaries would have to be pre-main-sequence objects; but we point out that Conti (1967) found 
no Lithium absorption in HD 118216. And it would also be useful to make a kinematical comparison between the Be stars and the RS CVn systems.

\section{Acknowledgements}

P. B. acknowledges support from the Deutsche Forschungsgemeinschaft and is very grateful to Drs L. Lucy and J. Ziółkowski for many inspiring discussions. D. S. H. acknowledges support from the Vanderbilt University Research Council.

\section{References}

Atkins, H. L. and Hall, D. S.: 1972, Publ. Astron. Soc. Pacific 84, 638.

Barnes, R. C.: 1974, Publ. Astron. Soc. Pacific 86, 195.

Biermann, P. and Tinsley, B. M.: 1974, Astron. Astrophys. 30, 1.

Bodenheimer, P. and Ostriker, J. P.: 1970, Astrophys. J. 161, 1101.

Catalano, S. and Rodono, M.: 1967, Mem. Soc. Astron. Italiana 38, 395.

Conti, P. S.: 1967, Astrophys. J. 149, 629.

Delhaye, J.: 1965, in A. Blaauw and M. Schmidt (eds), Galactic Structure, University of Chicago Press, p. 61.

Dworak, T. Z.: 1973, IAU Inform. Bull. Var. Stars No. 846.

Field, J. V.: 1969, Monthly Notices Roy. Astron. Soc. 144, 419.

Fricke, K. J.: 1968, Z. Astrophys. 68, 317.

Fricke, K. J. and Kippenhahn, R.: 1972, Ann. Rev. Astron. Astrophys. 10, 45.

Goldreich, P. and Schubert, G.: 1967, Astrophys. J. 150, 571.

Hall, D. S.: 1968, Publ. Astron. Soc. Pacific 80, 477.

Hall, D. S.: 1972, Publ. Astron. Soc. Pacific 84, 323.

Hall, D. S.: 1974, Acta Astron. 24, 215.

Hall, D. S.: 1975b, Acta Astron. (submitted).

Hall, D. S.: 1975a, Proc. IAU Colloq. No. 29 (in preparation).

Huang, S. S.: 1973, Bull. Am. Astron. Soc. 5, 41.

James, R. A. and Kahn, F. D.: 1970, Astron. Astrophys. 5, 232.

James, R. A. and Kahn, F. D.: 1971, Astron. Astrophys. 12, 332.

Kippenhahn, R.: 1969, Astron. Astrophys. 2, 309.

Kippenhahn, R., Meyer-Hoffmeister, E., and Thomas, H. C.: 1970, Astron. Astrophys. 5, 155.

Koch, R. H., Sobieski, S., and Wood, F. B.: 1963, Publ. Univ. Pennsylvania 9.

Kraft, R. P.: 1967, Publ. Astron. Soc. Pacific 79, 395.

Montle, R. E.: 1973, Masters Thesis, Vanderbilt University.

Oliver, J. P.: 1974, Ph.D. Thesis, University of California at Los Angeles.

Plavec, M. and Grygar J.: 1965, Kleine Veroeffentl. Remeis Sternw. Bamberg 4, No. 40, 213.

Roxburgh, I. W.: 1966a, Astron. J. 71, 133.

Roxburgh, I. W.: 1966b, Astrophys. J. 143, 111.

Ulrich, R. K. and Popper, D. M.: 1974, Bull. Am. Astron. Soc. 6, 461.

Wielen, R.: 1974, in G. Coutopoulos (ed.), Highlights of Astronomy, Vol. 3, D. Reidel, Dordrecht, p. 395 . 